\title{
Research on the Power System Fault Classification Based on HHT and SVM Using Wide-area Information
}

\author{
Yiran Guo, Changqing Li, Yali Li, Shibin Gao \\ Southwest Jiaotong University, Chengdu, China \\ Email: 779144969@qq.com
}

Received March, 2013

\begin{abstract}
A power system fault classification method based on the Hilbert-Huang transformation (HHT) and support vector machine (SVM) is proposed in this paper. According to different types of faults taking place in area and the outer area, this paper uses HHT to extract the instantaneous amplitude and Hilbert marginal spectrum of the current signal. Then a fault classifier consisting of a series of SVM classifiers that are optimized by using cross validation method is constructed. Finally, inputting the feature vector sets that are conversed by the HHT into the fault classifier, the fault type and locate the fault area will be distinguished. The simulation results show that this approach is very effective to classify the fault type especially when the sample is small.
\end{abstract}

Keywords: Power System; Fault Classification; HHT; SVM; Cross-validation

\section{Introduction}

An accurate diagnosis of grid faults type is very important to the stable operation of power networks. Wavelet analysis and wavelet packet analysis are used to extract feature vectors when dealing with the non-stationary signals that are generated when a fault occurs in a power system $[1,2]$. As a new signal processing means, HHT adopts Empirical Mode Decomposition (EMD), and HHT is applicable to analyze non-stationary signals. HHT has been successfully used in wave analysis, fault location and flicker measurement. There are some methods being used for classification, such as Neural Network (NN), SVM and so on [3-6]. When the sample is small, SVM will be a better choice with the higher accuracy rate and the better generalization ability $[7,8]$.

In this paper, the first step is to dissolve the original signals with EMD. And then instantaneous amplitude and marginal spectrum are extracted with HHT. A SVM multiple classifier is setup by integrating a series of SVM classifiers. And the last step is: inputting the instantaneous amplitude and marginal spectrum into the SVM multiple classifier whose parameters has been optimized with cross-validation methodology, then different types of power system faults will be distinguished.

\section{Fundamentals of HHT and SVM}

\subsection{Operating Principle of HHT}

HHT is made up of two parts: one part is to get IMF by handling signals with EMD, and another one is to get the time-frequency spectrum by handling IMF with Hilbert transform [9, 10]. Any signal can be broken down into some IMFs and a remainder function through handling it with EMD.

Through Hilbert transform, IMFs can be turned into as follows:

$$
y_{j}(t)=\frac{1}{\pi} \int_{-\infty}^{+\infty} \frac{I_{j}(\tau)}{t-\tau} d \tau
$$

$z_{j}(t)$ is composed of two parts: $y_{j}(t)$, the real part; and $I_{j}(t)$, the imaginary part. Meanwhile,

$$
z_{j}(t)=A_{j}(t) e^{i \theta_{j}(t)}
$$

where: $A_{j}$ is the instantaneous amplitude.

$$
\begin{aligned}
& A_{j}(t)=\sqrt{I_{j}^{2}(t)+y_{j}^{2}(t)} \\
& \theta_{j}(t)=\operatorname{arctg}\left(\frac{y_{j}(t)}{I_{j}(t)}\right)
\end{aligned}
$$

Depending on the definition of instantaneous frequency, the instantaneous frequency of IMF is:

$$
\omega_{j}(t)=\frac{d \theta_{j}(t)}{d t}
$$

After transforming all IMFs with Hilbert transform, a series of analytic functions and instantaneous frequencies 
can be got.

\subsection{Operating Principia of SVM}

Support vector machine (SVM) is a new method based on principles of statistics [11]. Its core is separating linearly separable problems by a hyperplane in the N-dimensional space.

It can be regarded as a convex quadratic program:

$$
\begin{array}{ll}
\min _{\omega, b} & \frac{1}{2}\|\vec{\omega}\|^{2} \\
\text { s.t. } & y_{i}((\vec{\omega} \bullet \vec{x})+b) \geq 0, i=1, \cdots, N
\end{array}
$$

and the quadratic programming problem can be rewritten as an optimization problem:

$$
\begin{array}{ll}
\min _{\alpha} & \\
\min _{\alpha} & \sum_{j=1}^{N} \alpha_{j}-\frac{1}{2} \sum_{i=1}^{N} \sum_{j=1}^{N} y_{i} y_{j}\left(x_{i} \bullet x_{j}\right) \alpha_{i} \alpha_{j} \\
\text { s.t. } & \sum_{i=1}^{N} y_{i} \alpha_{i}=0, C \geq \alpha_{i} \geq 0, i=1, \cdots, N
\end{array}
$$

when dividing nonlinear separable problem, the data should be mapped from the original space to high dimension feature space to make it linearly separable and the kernel function will be instructed to realize the data transformation in order to output the final classification results.

\section{Characteristic Quantity of Fault Current Signal}

To get the characteristic quantity of the current signal, first of all, fault time should be confirmed and then handling it with Hilbert-Huang transform. High-frequency signals will be generated when short-circuit fault occurs which reside in IMF1. So the instantaneous frequency of IMF1 will mutate immediately the short-circuit fault takes places. According to which the fault time can be judged.

1) Characteristic Quantity of the Instantaneous Amplitude

Through EMD decomposition, the original current signal can be changed into some IMFs. Choosing the IMF with the largest amplitude, and handling it with the HHT transform, the instantaneous amplitude feature can be got which can reflect the basic change rule of the original signal's amplitude.

For instantaneous amplitudes of the current of the same line, their range and changing rate will increase sharply after short circuit happens. So value $\mathrm{E}$ and standard deviation $\mathrm{X}$ are chosen as characteristic quantity of instantaneous amplitudes in order to present the changeable characteristics of breakdown signals.

2) Characteristic Quantity of the Marginal spectrum
High-frequency signals included in short-circuit will shunt through stray capacitance between lines or earth and lines. Purity of high-frequency signals will decrease with the distance increases. Its distribution is unequal in different phases. In this paper, high frequency signal levels(S) are chosen to be the characteristic quantity of the marginal spectrum.

\section{Constitution of SVM Classifier}

\subsection{Selection of Kernel Function and Parameter Optimization}

When structuring a SVM classifier, the kernel function should be chosen properly and optimized along with its relative parameters.

1) Selection of kernel function

There are two kinds of kernel functions. One is global kernel and another is local kernel. Local kernel is more suitable for classification problems. So Gaussian kernel function is chosen which is a typical local kernel of SVM classifier. And its form is as followed:

$$
K\left(\vec{x}, \overrightarrow{x_{i}}\right)=\exp \left(-\frac{\left\|\vec{x}-\overrightarrow{x_{i}}\right\|}{2 \sigma^{2}}\right)
$$

The final classification function is:

$$
f(\vec{x})=\operatorname{sgn}\left[\sum_{i=1}^{N} \alpha_{i} y_{i} \exp \left(-\frac{\left\|\vec{x}-\overrightarrow{x_{i}}\right\|}{2 \sigma^{2}}\right)+y_{i}-\sum_{i=1}^{N} y_{i} \alpha_{i}\left(x_{i} \bullet x_{j}\right)\right]
$$

where $C \geq \alpha_{i} \geq 0$

2) Parameter Optimization

Penalty factor $\mathrm{C}$ and kernel bandwidth do have great effects on the classification of a SVM classifier whose kernel function is Gaussian kernel function. Cross-validation methodology is selected to confirm this two parameters $[12,13]$. Sample data is divided into $\mathrm{N}$ sets, picking $\mathrm{N}-1$ sets as training data and the rest as testing data. So a series of average accuracy related to different parameters will be figured out. Then the set of parameter vector with the highest accuracy will be chosen as the very optimized parameter.

\subsection{The SVM Multiple Classifier}

A SVM multiple classifiers are made up by a series of SVM classifiers to distinguish all kinds of grid failures. Each SVM classifier can recognize one kind of grid failure with the output to be 1 or 0 to show what kind of grid failure happens. To distinguish four kinds of grid failures, four SVM classifiers is needed to compose a SVM multiple classifier.

\section{Detection of Fault Classification}

1) Extract current signal and get IMFs through EMD. 
Confirm the fault time by the instantaneous frequency of IMF1.

2) Deal with current signals measured in this nodal point or others next to it to figure out the characteristic quantity $\mathrm{E}, \mathrm{X}$ of the instantaneous amplitude and $\mathrm{S}$ of the marginal spectrum.

3) Optimize parameters of SVM classifiers with generalized cross validation.

4) Figure out support vectors to structure all kinds of SVM classifiers.

5) Gain the result by putting all characteristic quantity in the SVM multiple classifiers.

The whole process is shown in Figure 1.

\section{Simulation Verification}

Firstly, an IEEE-14 bus system is built with PSCAD, as is shown in Figure 2.

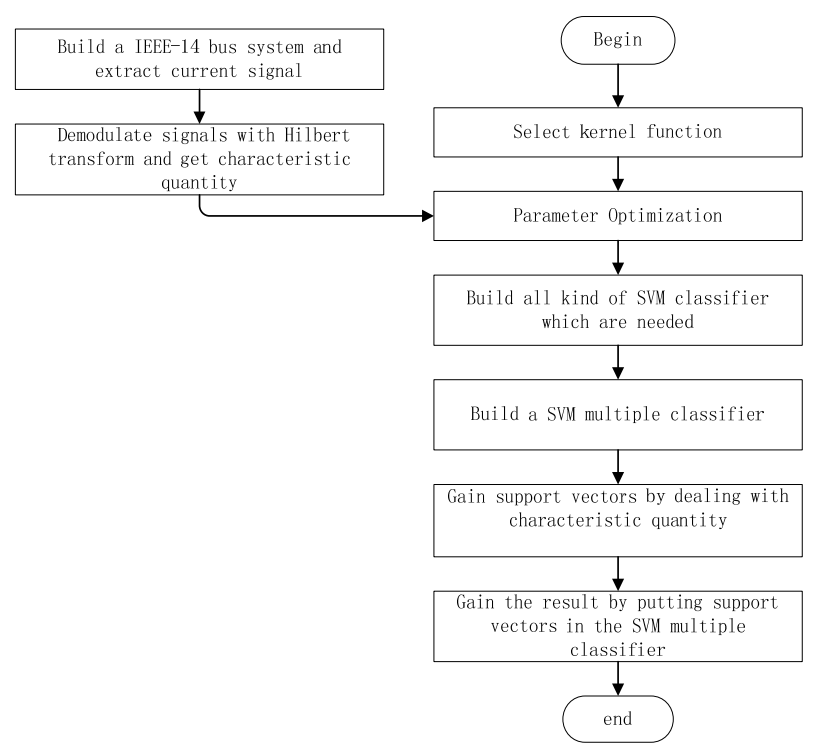

Figure 1. The calculation process of testing system fault.

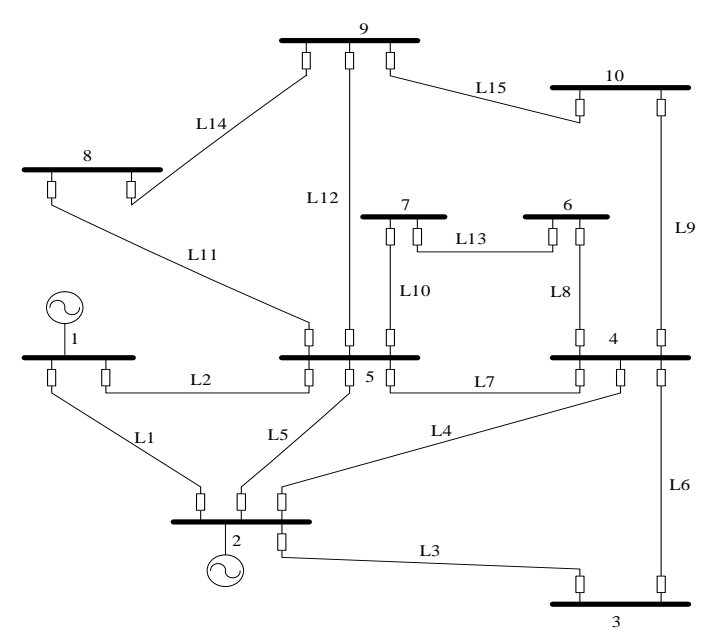

Figure 2. IEEE-14 bus system.
The length of line 15 is $180 \mathrm{~km}$ while the line 9 is 120 $\mathrm{km}$ long. Taking nodal point 9 as an example, different types of faults are set in line 15 and line 9, then three-phase current data of nodal points $8,9,5,10$ is recorded, finally analyzing the characteristic quantity and transfer them to node 9 .

Through simulation 54 sets of fault currents are obtained, taking 41 sets of them as the training sets and others as the test sets. For example, the single-phase fault happens on line 9, and the current waveform, instantaneous frequency, IMF, instantaneous amplitude, marginal spectrum will be shown in Figures 3-7.

Penalty factor $\mathrm{C}$ and kernel bandwidth are showed in Table 1.

The simulation results are showed in Table 2 .

In Table 2, a, b, c, d, e respectively represents single-phase short circuit, two-phase short circuit, twophase grounding fault, three-phase short circuit and normal operation. A being 1 means single-phase short circuit occurs in this area. A being -1 means that there's no single-phase short circuit occurring in this area. And the

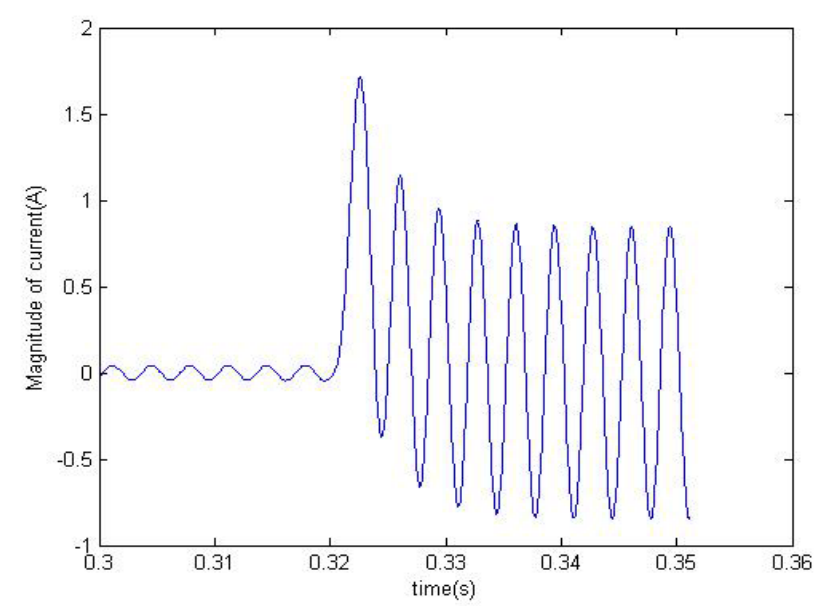

Figure 3. Fault current waveform.

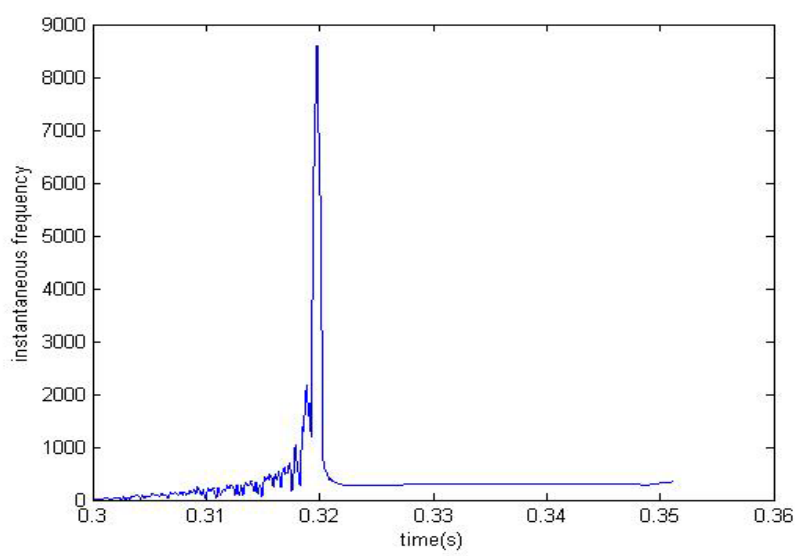

Figure 4. IMF1 instantaneous frequency of fault current. 

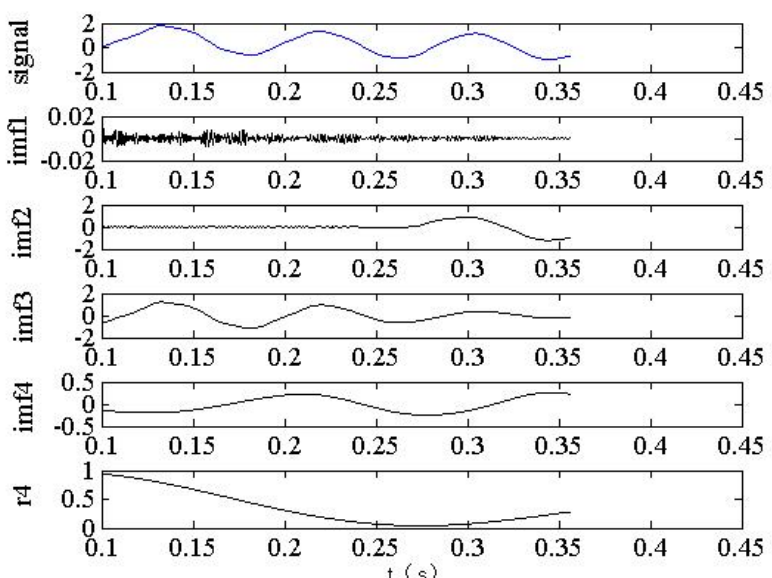

Figure 5. IMF signals of fault current.

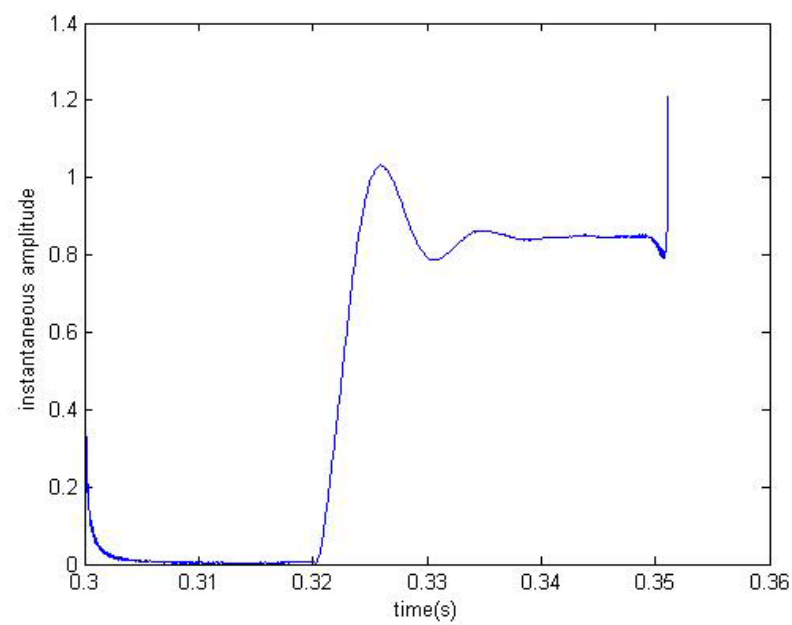

Figure 6. Instantaneous amplitude of IMF.

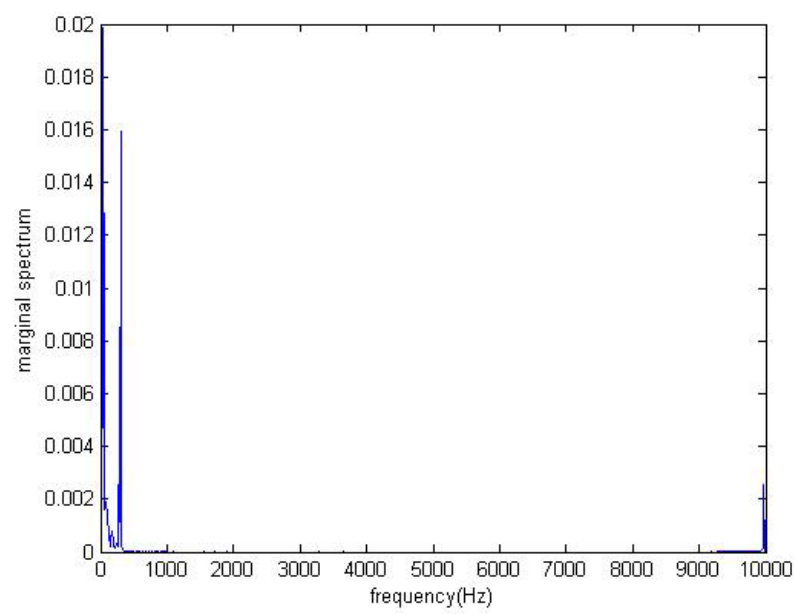

Figure 7. Hilbert marginal spectrum of fault current.

next step is using the SVM classifier and outputting the value of b. For example, (-1 -1 1 - - ) means two-phase grounding fault; ( -1 - $-1-1-1$ - 1 ) means external fault; ( -1
Table 1. SVM parameters.

\begin{tabular}{ccc}
\hline & penalty factor C & Kernel bandwidth $\sigma$ \\
\hline SVM1 & 69 & 17 \\
SVM2 & 82 & 17 \\
SVM3 & 90 & 93 \\
SVM4 & 109 & 84 \\
\hline
\end{tabular}

Table 2. Classification results of simulation experiments.

\begin{tabular}{|c|c|c|c|}
\hline $\begin{array}{c}\text { sequence } \\
\text { number }\end{array}$ & fault location & Fault type & Outcome \\
\hline 1 & \multirow{4}{*}{$\begin{array}{l}\text { About } 20 \mathrm{~km} \text { from } \\
\text { node } 9 \text { on L15 }\end{array}$} & One phase short circuit & $1-\cdots$ \\
\hline 2 & & Two-phase short circuit fault & $-11---$ \\
\hline 3 & & two-phase ground fault & $-1-11--$ \\
\hline 4 & & bolted three-phase fault & $-1-1-11-$ \\
\hline 5 & \multirow{4}{*}{$\begin{array}{l}\text { About } 70 \mathrm{~km} \text { from } \\
\text { node } 9 \text { on L15 }\end{array}$} & one-phase short-circuit & $1---$ \\
\hline 6 & & Two-phase short circuit fault & $-11---$ \\
\hline 7 & & two-phase ground fault & $-1-11--$ \\
\hline 8 & & bolted three-phase fault & $-1-1-11-$ \\
\hline 9 & \multirow{4}{*}{$\begin{array}{l}\text { About } 40 \mathrm{~km} \text { from } \\
\text { node } 10 \text { on L9 } \\
\text { (outside the } \\
\text { region) }\end{array}$} & one-phase short-circuit & $-1-1-1-11$ \\
\hline 10 & & Two-phase short circuit fault & $-1-1-1-11$ \\
\hline 11 & & two-phase ground fault & $-1-1-1-11$ \\
\hline 12 & & bolted three-phase fault & $-1-1-1-11$ \\
\hline 13 & trouble-free & trouble-free & $\begin{array}{c}-1-1-1-1 \\
-1\end{array}$ \\
\hline
\end{tabular}

-1 -1 - - -1) means normal operation. Table 2 shows that the results of 13 sets of tests are right.

\section{Conclusions}

In this paper, HHT and SVM are adopted to distinguish different types of power system faults. Downtime is determined by using HHT. Through analysis of the fault current, instantaneous amplitude and marginal spectrum are defined to characterize electric current fluctuation characteristic. The method of cross-validation is used to optimize the parameters of SVM classifiers. Then a SVM multiple classifier is set up using wide-area information to test power system faults by taking advantage of SVM's abilities of its self learning and dealing with small samples. And the simulation results show that this approach can distinguish fault types of power supply line with a high accuracy.

\section{REFERENCES}

[1] X. Z. Yi and X. Q. Li, "Diagnosis of Power Supply Line Fault Based on Wavelet Analysis and Support Vector Machine,” Journal of Beijing institute of Petro-chemical 
Technology, Vol. 13, No. 3, 2005, pp. 36-40.

[2] Y. H. Liu, Y. Y. Wang and Z. H. Song, “Adaptive Fault Feature Extraction Based on Stationary Wavelet Packet Decomposition and Hilbert Transform," Transacions of China Electrotechnical Society, Vol. 24, No. 2, 2009, pp. 145-149.

[3] X. L. Zhang, X. J. Zeng, H. J. Ma, et al., "Power Grid Faults Location with Traveling Wave Based on Hilbert-Huang Transform," Automation of electric power systems, Vol. 32, No. 8, 2008, pp. 64-67

[4] S. Han, L. Q. He, B. Sun, et al., "Hilbert-Huang Transform Based Nonlinear and Non-Stationary Analysis of Power System Low Frequency Oscillation and Its Application,” Power System Technology, Vol. 32, No. 4, 2008, pp. 56-60.

[5] Y. X. Su, Z. G. Liu, K. L. Li, et al., "Application of Hilbert-Huang Transform in Harmonic Detection of Electrified Railway,” Power System Technology, Vol. 32, No. 18, 2008, pp. 30-35

[6] Z. J. Kang, L. Xu, J. C. Fan, et al., "Fault Location for High Voltage Transmission Lines With the Series Compensation Capacitor Based on Hilbert-Huang Transform and Neural Network," Power System Technology, Vol. 33, No. 20, 2009, pp. 142-146

[7] J. Manikandan and B. Venkataramani, "Design of a Modified One-against-all SVM Classifier,” Proceedings of the 2009 IEEE International Conference on Systems, man, and cybernetics, 2009, pp. 1869-1874

[8] D. C. Yang, C. Rehtanz, Y. Li, W. Tang and R. Q. Qu, "Researching on Low Frequency Oscillation in Power System Based on Improved HHT Algorithm," Proceedings of the CSEE, Vol. 31, No.10, 2011, pp.102-108.

[9] H. Jiang, X. Q. Wang and J. C. Peng, "Method to Measure Voltage Flicker Based on Hilbert-Huang Transform,” Power System Technology, Vol. 35, No. 9, 2012, pp. 250-256.

[10] Q. Zhang and Y. Q. Yang, "Research of the Kernel Fuction of Support Vector Machine,” Electric Power Science and Engineering, Vol. 28, No. 5, 2012, pp. 42-45.

[11] T. Y. Li, C. L. Chen, W. L. Cheng, et al., "Application of cross Validation in Power Quality Denoisin,” Automation of Electric Power Systems, Vol. 319, No. 16, 2007, pp. 75-78.

[12] J. Ma, X.Wang and Z. P. Wang, "A New Fault Phase Identification Method Based on Phase Current Difference," Proceedings of the CSEE, Vol. 31, No. 10, 2011, pp. 102-108

[13] Y. Wang, Y. X. Zhang and S. X. Xu, "Fault Location and Phase Selection for Parallel Transmission Lines Based on Wide Area Measurement System,” Automation of Electric Power Systems, Vol. 34, No. 6, 2010, pp. 65-69. 